\title{
Intelligent Robust Controller Design for a Micro-Actuator
}

\author{
Marialena Vagia, George Nikolakopoulos and Anthony Tzes
}

\begin{abstract}
In this article the design of an intelligent robust controller for a Micro-Actuator $(\mu-A)$ is presented. The $\mu-A$ is composed of a micro-capacitor, whose one plate is clamped while its other flexible plate's motion is constrained by hinges acting as a combination of springs and dashpots. The distance of the plates is varied by the applied voltage between them. The dynamics of the plate's rigid-body motion results in an unstable, nonlinear system. The control structure is constructed from: a) a feedforward controller which stabilizes the microactuator around its nominal operating point, b) a robust PID controller whose gains are initially tuned via the utilization of Linear Matrix Inequalities (LMIs) and subsequently are switched according to the position of the upper plate, and c) an intelligent prefilter which appropriately shapes the reference signal. The resulting overall control scheme is applied to the non-linear model of the $\mu-\mathrm{A}$ where simulation results are presented to prove the efficacy of the suggested scheme.
\end{abstract}

\section{INTRODUCTION}

Micro-devices in the form of actuators and sensors are becoming increasingly dominant nowadays $[1,2]$ with the technological improvements in the area of micro-fabrications [3] setting new limits in the design of micro-devices. In the area of micro-actuators, several micro-structures $[4,5]$ have been used as the primitive components. These actuators could be utilized in many applications where the application of a force (picoNewtons), in conjunction with their positioning, is needed [6-8].

The utilization of modern $\mu-\mathrm{A}$ demands new approaches regarding the control of these structures. Due to their diminution, there is a need to utilize advance control techniques for satisfying certain performance objectives [9]. These techniques primary stem from the modelling peculiarities of these devices as many factors that are ignored in the macro-world play an important role in the micro-world. Moreover the non-linearity of the $\mu-\mathrm{A}$ model is forcing the control designer to linearize the model before applying the advance control techniques instead of relying on the design of non-linear controllers, which are very complicated to be implemented.

For the resulting multiple linearized models of the $\mu-$ actuator a combination of advanced control techniques and appropriate pre-filtering seems to be essential for a robust and high-quality control of this demanding $\mu$-actuators.

In this article an LMI-based robust PID controller is designed and applied for controlling the positioning of a $\mu-$ capacitor's plate. This control design approach is based on: a)

This work was partially funded by the European Social Fund (ESF), Operational Program for Educational and Vocational Training II (EPEAEK II) and particularly the Program HRAKLEITOS, No. B238.012.

The authors are with the Electrical and Computer Engineering Department, University of Patras, 26500 Rio Achaia, Greece. Corresponding author's e-mail tzes@ee.upatras.gr a feedforward controller that aims to stabilize the capacitor's plate around the selected nominal point of operation, b) a tuning process for the utilized LMIs for computing the PIDcontrol action, and c) an intelligent-tuned prefiltering process for shaping appropriately the reference signal prior to the controller excitation.

In the rest of this article the development of the $\mu-A$ model is carried in Section II, while the design of the controller is presented in Section III. In Section IV extended simulation studies that prove the efficacy of the proposed control scheme are presented. Finally in Section $\mathrm{V}$ the conclusions are drawn.

\section{Micro-Actuator Modelling}

The $\mu$-A corresponds to a micro-capacitor whose one plate is attached to the ground while its other moving plate is floating in air. The boundary of the moving plate is either supported (pinned) or constrained by hinges (springs), as shown in Figure 1.

\section{A. Dynamic Plate Modelling}

The equation of motion for a 2-D distributed thin plate [10] floating on air and supported at its boundary is expressed as follows

$$
\mathcal{L} w(x, y, t)+\mathcal{C} \dot{w}(x, y, t)+m_{p} \ddot{w}(x, y, t)=f(x, y, t),
$$

where $\mathcal{L}$ is a time-invariant, symmetric, non-negative differential operator, $\mathcal{C}$ is a structural damping operator $\mathcal{C}=$ $\alpha_{1}+\alpha_{2} m_{p}, m_{p}$ is the mass density of the 2-D structure, $f(x, y, t)$ is the time-varying distributed control force acting on the thin plate at the $(x, y)$-coordinate.

In thin plate theory the operator $\mathcal{L} w(x, y, t)$ is

$$
\frac{E h^{3}}{12\left(1-\nu^{2}\right)}\left(w_{x x x x}+2 w_{x x y y}+w_{y y y y}\right),
$$

where $E$ is the Young's modulus, $\nu$ is the Poisson's ration of the plate material, $h$ is the thickness of the plate, and the symbol $w_{x y}$ corresponds to $\frac{\partial}{\partial x} \frac{\partial}{\partial y} w(x, y, t)$.

For a thin square plate of length $\ell$, shown in Figure 1, the equations of motion along with its boundary conditions are:

$$
\begin{aligned}
& \begin{array}{c}
w_{t t}+D_{1}\left(w_{x x x x}+2 w_{x x y y}+w_{y y y y}\right)=0, \\
0<x, y<\ell, t>0
\end{array} \\
& \begin{array}{c}
w(x, y, 0)=w_{0}(x, y), \quad w_{t}(x, y, 0)=w_{1}(x, y), \\
0<x, y<\ell,
\end{array} \\
& \begin{array}{r}
w(0, y, t)=w(\ell, y, t)=w_{x x}(0, y, t)=w_{x x}(\ell, y, t)=0, \\
0<y<\ell
\end{array} \\
& \begin{array}{l}
D\left(w_{y y y}+(2-\nu) w_{x x y}\right)=-k^{2} w, y=0,0<x<\ell \\
D\left(w_{y y y}+(2-\nu) w_{x x y}\right)=k^{2} w, y=\ell, 0<x<\ell \\
w_{y y}+\nu w_{x x}=0, y=0, y=\ell, 0<x<\ell
\end{array}
\end{aligned}
$$




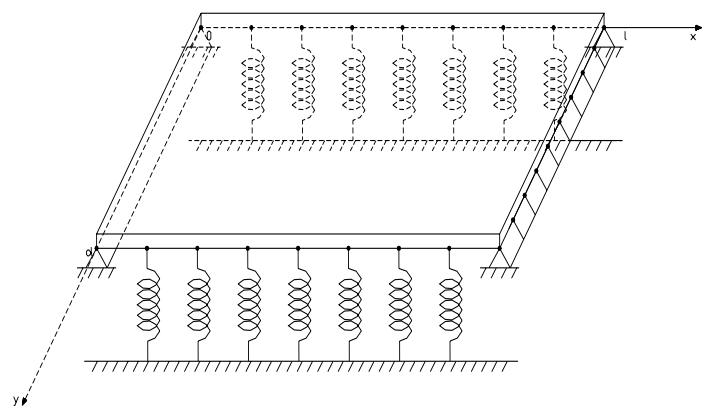

Fig. 1. $\mu$ - Actuator Schematic

where $D_{1}=\frac{D}{\rho}=\frac{E h^{3}}{12 m_{p}\left(1-\nu^{2}\right)}, w_{0}(x, y)\left(w_{1}(x, y)\right)$ is the initial displacement (velocity) of the plate in $z$-direction, and $k$ represents the linear restoring force of the springs.

Application of the assumed modes method presumes that the displacement and point control force are expressed as

$$
\begin{aligned}
w(x, y, t) & =\sum_{i=1}^{\infty} W_{i}(x, y) \eta_{i}(t) \\
f(x, y, t) & =\sum_{i=1}^{p} F_{i}(t) \delta\left(x-x_{i}\right) \delta\left(y-y_{i}\right)
\end{aligned}
$$

where $\eta_{i}(t)$ is the $i$-th mode modal displacement, $F_{i}(t)$ is the force amplitude, $p$ is the number of actuators, $\delta\left(x-x_{i}\right)$ and $\delta\left(y-y_{i}\right)$ are spatial Dirac delta functions.

For the given stated boundary conditions, closed form solutions can be found [11] for the free-response expressions $w(x, y, t)$. Retaining a finite number of modes the ordinary differential equation describing the motion for the $n$-th mode is

$$
\ddot{\eta}_{n}+\left(\alpha_{1} \omega_{n}^{2}+\alpha_{2}\right) \dot{\eta}_{n}+\omega_{n}^{2} \eta_{n}=\sum_{i=1}^{p} W_{n}^{*}\left(x_{i}, y_{i}\right) F_{i} .
$$

When: 1) the forcing element $f(x, y, t)=f(t)$ is independent of the point of application, 2) there is no proportional damping $\left(\alpha_{1}=0\right)$, and 3$)$ retaining only one mode $(n=1)$ the equation of motion degenerates to

$$
\ddot{\eta}_{1}+\alpha_{2} \dot{\eta}_{1}+\omega_{1}^{2} \eta_{1}=W_{1}^{*} F .
$$

The resulting movement produced by this mode is displayed in Figure 2. In this case, the displacement of the plate $z(t)=$

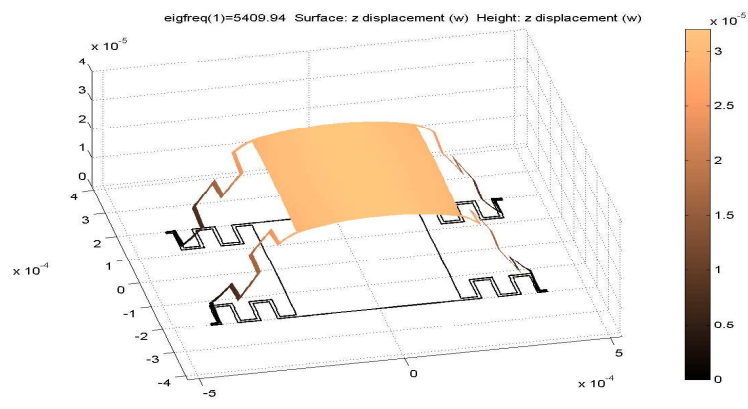

Fig. 2. $\mu$-Actuator's 1st-Vibrational mode

$w(x, y, t)$ is identical for all points $(x, y)$ of the plate and equal to $\eta_{1}(t)$. Multiplication of both sides of ( 1$)$ by $W_{1}^{*}=$ $m$ yields the following equation of motion $m \ddot{\eta}_{1}+b \dot{\eta}_{1}+k \eta_{1}=$ $F$, where $m$ is the total mass of the plate, and $k$ is the overall stiffness of the springs, as shown in Figure 3.

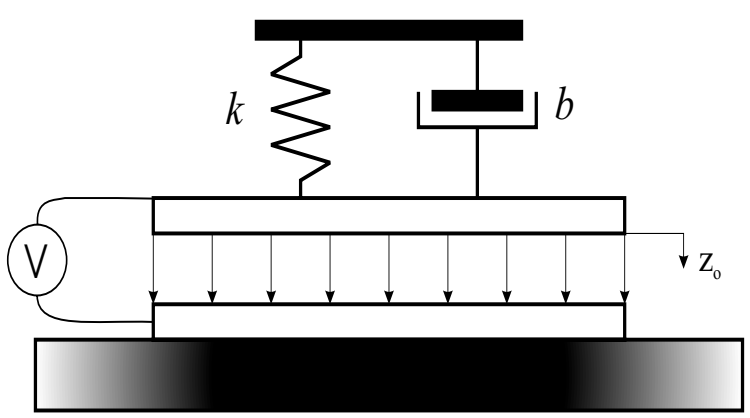

Fig. 3. Rigid body dynamical modelling

\section{B. Electrical Force Model}

Application of a voltage $U$ between the capacitor's plates generates an electrically-induced force

$$
F=\frac{\varepsilon A U^{2}}{2 s^{2}},
$$

where $A$ is the area of the plates, $\epsilon$ is the dielectricic constant and $s$ is the distance between the plates when the spring is relaxed.

The nonlinear equation of motion for the displacement $x$ from the equilibrium point is given by [12]

$$
m \ddot{\eta}_{1}+b \dot{\eta}_{1}+k \eta_{1}=\frac{a U^{2}}{\left(s-\eta_{1}\right)^{2}}
$$

and $a=\frac{\varepsilon A}{2}$.

The "equilibria"-points $\eta_{1, i}^{o}, i=1, \ldots, M$ depend on the applied nominal voltages $U_{o}$. Equation (2) for $\dot{\eta}_{1, i}^{o}=0$ yields

$$
U_{o}= \pm\left[\frac{k \eta_{1, i}^{o}\left(s-\eta_{1, i}^{o}\right)^{2}}{a}\right]^{1 / 2}
$$

This nominal $U_{o}$-voltage must be applied if the capacitor's plate is to be maintained at a distance $\eta_{1, i}^{o}$ from its unstretched position.

The linearized equations of motion around the equilibria points $\left(U_{o}, \eta_{1, i}^{o}, \dot{\eta}_{1, i}^{o}=0\right)$ can be found using standard perturbation theory for the variables $U$ and $\eta_{1}$ where $U=$ $U_{o}+\delta u$ and $\eta_{1, i}=\eta_{1, i}^{o}+\delta \eta_{1, i}$. The equation of motion for the perturbed system is:

$$
\begin{aligned}
m \delta \ddot{\eta}_{1, i}+b \delta \dot{\eta}_{1, i}+ & k \eta_{1, i}^{o}+k \delta \eta_{1, i}=\frac{a U_{o}^{2}}{\left(s-\eta_{1, i}^{o}\right)^{2}} \\
& +\frac{2 a U_{o}^{2}}{\left(s-\eta_{1, i}^{o}\right)^{3}} \delta \eta_{1}+\frac{2 a U_{o}}{\left(s-\eta_{1, i}^{o}\right)^{2}} \delta u .
\end{aligned}
$$

Inserting the expression from (3) into (4) we obtain

$$
m \delta \ddot{\eta}_{1, i}+b \delta \dot{\eta}_{1, i}+K_{i} \delta \eta_{1}=\beta_{i} \delta u
$$


where $K_{i}=\left[k-\frac{2 a U_{o}^{2}}{\left(s-\eta_{1, i}^{o}\right)^{3}}\right]$, and $\beta_{i}=\left[\frac{2 a U_{o}}{\left(s-\eta_{1, i}^{o}\right)^{2}}\right]$. The state space description of (5) is

$$
\begin{aligned}
{\left[\begin{array}{l}
\delta \dot{\eta}_{1, i} \\
\delta \ddot{\eta}_{1, i}
\end{array}\right] } & =\left[\begin{array}{cc}
0 & 1 \\
-\frac{K_{i}}{m} & -\frac{b}{m}
\end{array}\right]\left[\begin{array}{c}
\delta \eta_{1, i} \\
\delta \dot{\eta}_{1, i}
\end{array}\right]+\left[\begin{array}{c}
0 \\
\frac{\beta_{i}}{m}
\end{array}\right] \delta u(6) \\
& =\tilde{A}_{i}\left[\begin{array}{l}
\delta \eta_{1, i} \\
\delta \dot{\eta}_{1, i}
\end{array}\right]+B_{i} \delta u, \quad i=1, \ldots, M .
\end{aligned}
$$

\section{INTElligent CONTROLlER DESIGN}

The proposed controller is a combined robust PID controller [13] with a Feedforward Controller (FC) and an Intelligent Prefilter as shown in Figure 4. The lowpass

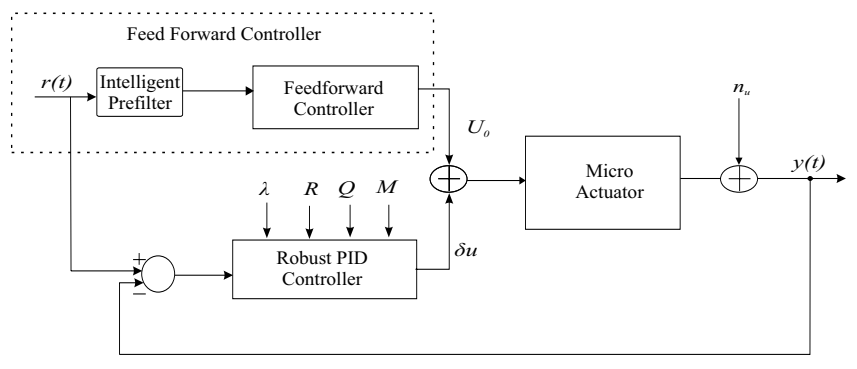

Fig. 4. LMI-based Robust PID Control Architecture

prefilter acts on the reference signal; its cut-off frequency is tuned according to the spectrum of the reference signal. The FC-term provides the $U_{o}$ portion in (3).

\section{A. Robust PID-Controller Design}

The feedback term is a robust PID controller for the set of the $M$-linearized systems in (7) and is tuned with the utilization of LMIs [14] and a design procedure that is based on the theory of Linear Quadratic Regulators (LQR).

This robust PID controller is specially designed to address the case where multiple-system models [15-17] have been utilized in order to describe the uncertainties that are inherent from the linearization process of the non-linear system model.

The nature of a PID-structure in the controller design can be achieved if the linearized system's state vector $\bar{\eta}=\left[\delta \eta_{1, i}, \delta \dot{\eta}_{1, i}\right]^{T}$ is augmented with the integral of the error signal $\int e d t=\int\left(r(t)-\eta_{1, i}(t)\right) d t$. In this case, the augmented system's description is

$$
\left[\begin{array}{c}
\dot{\bar{\eta}} \\
-e
\end{array}\right]=\hat{A}_{i}\left[\begin{array}{c}
\bar{\eta} \\
-\int^{2} e d t
\end{array}\right]+\left[\begin{array}{c}
B_{i} \\
0
\end{array}\right] \delta u+\left[\begin{array}{c}
-1 \\
0
\end{array}\right] r
$$

where $\hat{A}_{i}=\left[\begin{array}{cc}\tilde{A}_{i} & 0 \\ 1 & 0\end{array}\right]$.

The LQR-problem for the systems described in (8) can be cast in the computation of $\delta u$ in order to minimize the following cost:

$$
J(\delta u)=\int_{0}^{\infty}\left(\tilde{\eta}^{T} Q \tilde{\eta}+\delta u^{T} R \delta u\right) d t
$$

where $\tilde{\eta}=\left[\bar{\eta},-\int e d t\right]^{T}$, and $Q, R$ are semidefinite and definite matrices respectively. Rather than using the $\hat{A}_{i-}$ matrices in the LQR-problem, the introduction of the auxiliaries matrices $A_{i}=\hat{A}_{i}+\Lambda \mathbf{I}$, where $\Lambda>0$ and $\mathbf{I}$ the identity matrix generates an optimal control $\delta u=-S \bar{\eta}$ such that the closed-loop's poles have real part less than $-\lambda$, or $\Re\left(\operatorname{eig}\left(\hat{A}_{i}-B S\right)\right)<-\Lambda \forall i \in\{1, \ldots, M\}$.

The optimal control $\delta u=-S \tilde{\eta}$ can be computed via the following LMI-based algorithm, where a set of auxiliary matrices $\hat{P}, Y$ and an additional variable $\gamma(\gamma>0)$ have been introduced.

1) Start with a large value of $\gamma$.

2) Compute a feasible solution $\hat{P}, Y$ (if exists) to the following set of convex constraints:

$$
\left[\begin{array}{cc}
\gamma & \tilde{\eta}^{T}(0) \\
\tilde{\eta}(0) & \hat{P}
\end{array}\right] \leq 0
$$

$$
\text { For } i=1, \ldots, M
$$

$$
\left[\begin{array}{ccc}
A_{i} \hat{P}+\hat{P} A_{i}^{T}+B_{i} Y+Y^{T} B_{i}^{T} & \hat{P} & Y^{T} \\
\hat{P} & -Q^{-1} & 0 \\
Y & 0 & -R^{-1}
\end{array}\right] \leq 0
$$

3) Reduce $\gamma$ by $\delta \gamma(\gamma-\delta \gamma \rightarrow \gamma)$ and Return to Step 1 . The feedback control can be computed based on the recorded values of $\hat{P}^{*}$ and $Y^{*}$ for the last feasible solution:

$$
\begin{aligned}
& \delta u=Y^{*}\left(\hat{P}^{*}\right)^{-1} \tilde{\eta}=-S \tilde{\eta}=-S\left[\begin{array}{c}
\bar{\eta} \\
-\int e d t
\end{array}\right] \\
& =-\left[s_{p}\left|s_{d}\right| s_{i}\right]\left[\begin{array}{c}
\delta \eta_{1, i} \\
\delta \dot{\eta}_{1, i} \\
-\int e d t
\end{array}\right] \\
& =\left[s_{p} e+s_{d} \dot{e}+s_{i} \int e d t\right]+\left[s_{p}\left(\eta_{1, i}^{o}-r\right)-s_{d} \dot{r}\right] .
\end{aligned}
$$

The first portion of the controller form in (14) is equivalent to that of a PID-controller.

\section{B. Intelligent Prefilter Design}

The scope of the Intelligent Preshaping Filter (IPF) is to suppress the high harmonics of the excitation signal, particularly in the case of high speed maneuvers. This is due to the nonlinear (quadratic) nature of the system in (2), where second harmonic resonances can be developed. These second harmonic resonances can de generated either from the output of the PID-controller, or from the feedforward term. Indirectly, the output from both of these portions is influenced from the spectrum content of the reference signal $r(t)$.

A simple first order lowpass filter of the form $G_{I F}=$ $\frac{p}{s+p}$ is used for appropriately shaping the reference signal, as shown in Figure 5. The selection of the cutoff frequency " $p$ " is made in order to: a) extend the robustness of the system towards large variations of the spectrum content of the reference signal, and b) decrease the response time of the system in small maneuvers. These two objectives are in conflict since for the first case a reduction of the filter bandwidth of the system is needed while for the second case an increase of the filter bandwidth is necessary for decreasing the system's response time.

In this research effort, the $p$-parameter is tuned based on an intelligent ad-hoc procedure. 
The spectrum content of the reference signal can be captured through the usage of the Fourier transform. Under the assumption that the reference signal $r(t)$ is sampled with a period of $T_{s}$, its harmonic content over the last $N$-samples $\left(r\left(t-(N-1) T_{s}\right), \ldots, r\left(t-T_{s}\right), r(t)\right)$ can be computed via the Discrete Fourier Transform. Let the frequency transformed signal over the sliding window $N T_{s}$ at time $t$ be $\mathcal{R}_{t}(\omega), \omega \in \Delta \omega\{0, \ldots,(N-1)\}$, where $\Delta \omega=\frac{2 \pi}{N T_{s}} ;$ an index describing variations of spectrum content between different time instants $t^{\prime}$ and $t$ can be of the form

$$
I_{s c}=\sum_{j=0}^{\frac{N}{2}}\left|\mathcal{R}_{t^{\prime}}(j \Delta \omega)-\mathcal{R}_{t}(j \Delta \omega)\right|
$$

Large values of $I_{s c}$ indicate a dissimilarity between $\mathcal{R}_{t^{\prime}}(\omega)$ and $\mathcal{R}_{t}(\omega)$.

Similarly the notion of a "small maneuver" can be captured by the difference

$$
I_{s m}=\left|r\left(t^{\prime}\right)-r(t)\right| \text {. }
$$

From the aforementioned discussion, the $p$-cutoff frequency should be inverse proportional to the $I_{s c}$ and $I_{s m}$, or

$$
p=\frac{1}{\xi_{1} I_{s c}+\xi_{2} I_{s m}},
$$

where $\xi_{1}, \xi_{2} \geq 0$.

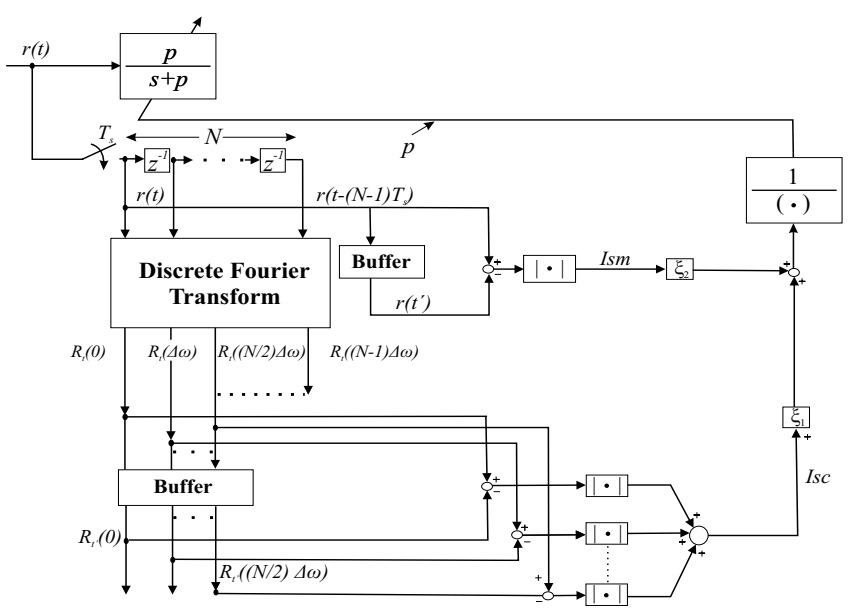

Fig. 5. Tuning Scheme of Intelligent Prefilter

\section{Simulation Studies}

Simulation studies were carried on a $\mu$-A's non-linear model, whose its $\mathrm{SiO}_{2}$-plates have an area $A=400 \mu \mathrm{m}$ $\times 400 \mu \mathrm{m}$, with a mass $m=7.0496 \cdot 10^{-10} \mathrm{Kgr}$. The initial gap was set to $s=4 \mu \mathrm{m}$ while the dielectric constant of the air was $\varepsilon=9 \cdot 10^{-12} \frac{\text { Coulomb }^{2}}{N \cdot m^{2}}$. The allowable displacements of the micro-capacitor's plate in the vertical axis were $\eta_{1} \in[0.1,3.9] \mu \mathrm{m}$. The goal of the controller was to move the capacitor's plates from an initial position to a new desired one (set-point regulation). Two distinct cases, as far as the number of "equilibria" points were examined:
1) $M=1$ and $\eta_{1,1}^{o}=1.9 \mu \mathrm{m}$, and 2) $M=10$ and $\eta_{1, i}^{o}=0.1+\frac{3.9-0.1}{2 \cdot 10}+(i-1) 0.38 \mu \mathrm{m}, i=1, \ldots, 10$. Each linearized system is valid in only one of these $M$-regions (i.e., for the second case, the first subsystem with $\eta_{1,1}^{o}=0.29$ is valid for all values of $\eta_{1}$ within $\left.[0.1,0.48) \mu \mathrm{m}\right)$.

The proposed control scheme has been applied in multiple simulation test cases in order to test its efficacy. Particular attention has been paid in order to identify any relevance among the $M$-number of the operating points and the performance of the controller on the nonlinear system. In the sequel, unless otherwise stated the parameters used in the formulation of the LQR-cost were $R=10^{-8}$ and $Q=10^{-6} \mathbf{I}_{3 \times 3}$. Similarly, the measurements are assumed to be corrupted with noise of level such that the $S N R=20 \mathrm{db}$. The cutoff of the filter $p$ could float in the range between 200 and $2000 \frac{\mathrm{rad}}{\mathrm{sec}}$; the parameters used for its adjustment were $\xi_{1}=0.05, \xi_{2}=0.95, T_{s}=10 \mathrm{nsec}$, and $N=128$ samples. Since the $I_{s m}$ dominates the adjustment of the filter's cutoff, Figure 6 presents in a graphical manner the relationship between $p$ and $I_{s m}$. The micro-actuator while

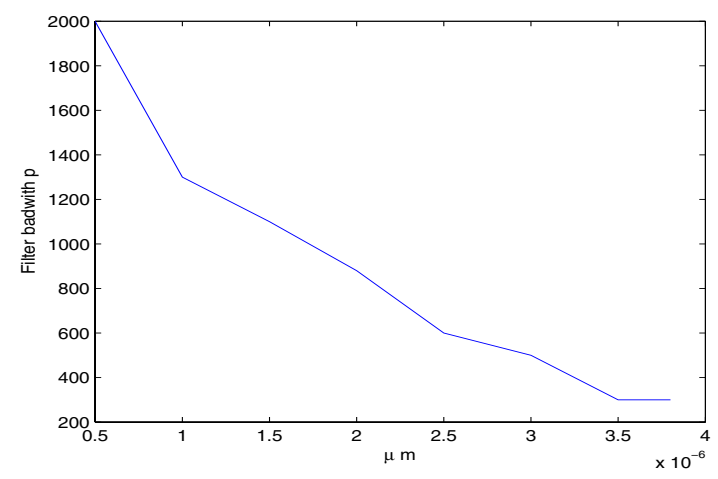

Fig. 6. Dependence between $p$-parameter and displacement $I_{s m}$

at rest at $0.1 \mu \mathrm{m}\left(\eta_{1}(0)=0.1 \mu \mathrm{m}\right)$ is excited with a step signal of amplitude $2.4 \times 10^{6}$ at time 0.02 seconds.

In Figure 7 we present the responses of the microcapacitor for $M=1$ and two cases of "prescribed" stability, namely i) $\Lambda=1$, and ii) $\Lambda=20$. As expected, the system responds faster in the second case since it is guaranteed that its closed-loop poles will have real part less than -20 . The control effort is presented in Figures 8 and 9 for the first and second case, respectively.

For the case of 10-operating points the step responses of the micro-capacitor and the relevant control effort are displayed in Figures 10, 11, 12 respectively. Comparing the responses in Figures 7 and 10, an apparent performance improvement is observed when using more operating points. However, due to the continuous switching between the operating regimes, the control effort in the latter case $(M=10)$ is quite "noisy" (i.e., compare the recorded values of $U(t)$ in Figures 8 and 11) and can cause significant aging on the micro-capacitor plate. On the other hand the control signal seems to have less overshoot in the case where more operating points are used. 


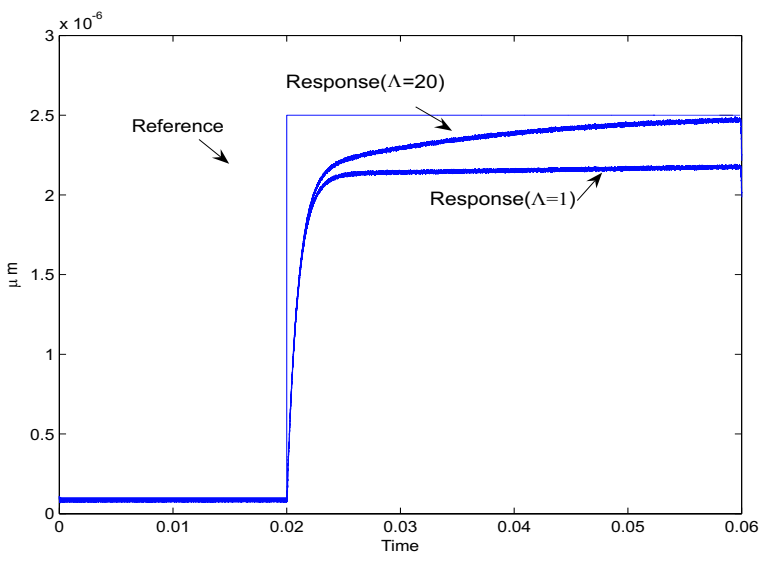

Fig. 7. Micro-actuator step response ( $M=1, \Lambda=1$ and $\Lambda=20$ cases)

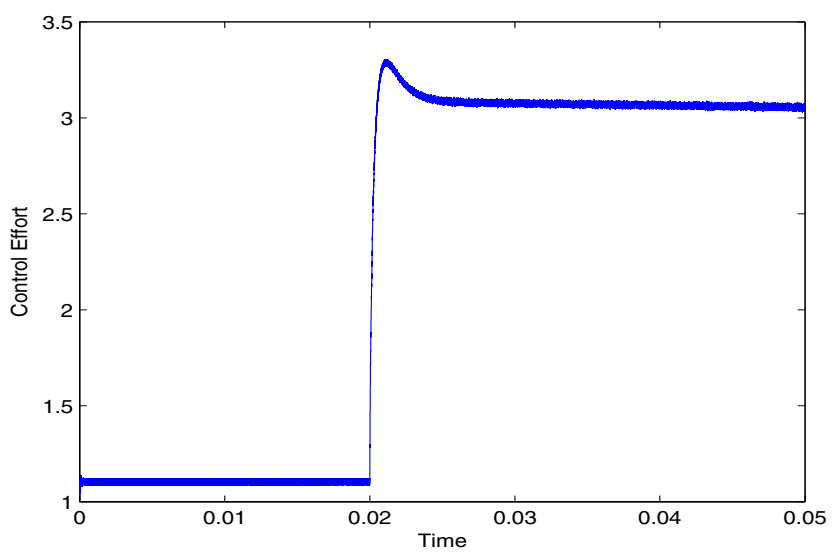

Fig. 8. Controller effort (1-operating point, $\Lambda=1$ )

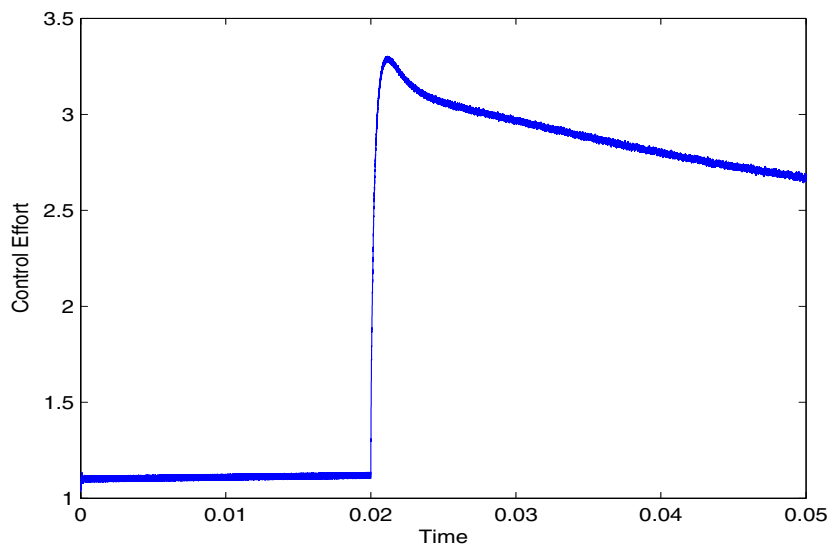

Fig. 9. Controller effort (1-operating point, $\Lambda=20$ )

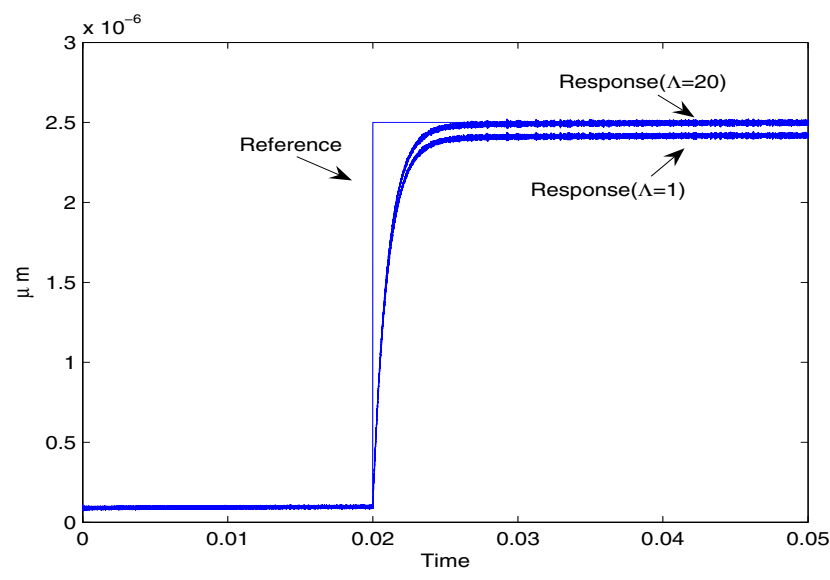

Fig. 10. Micro-actuator step response $(M=10, \Lambda=1$ and $\Lambda=20$ cases)

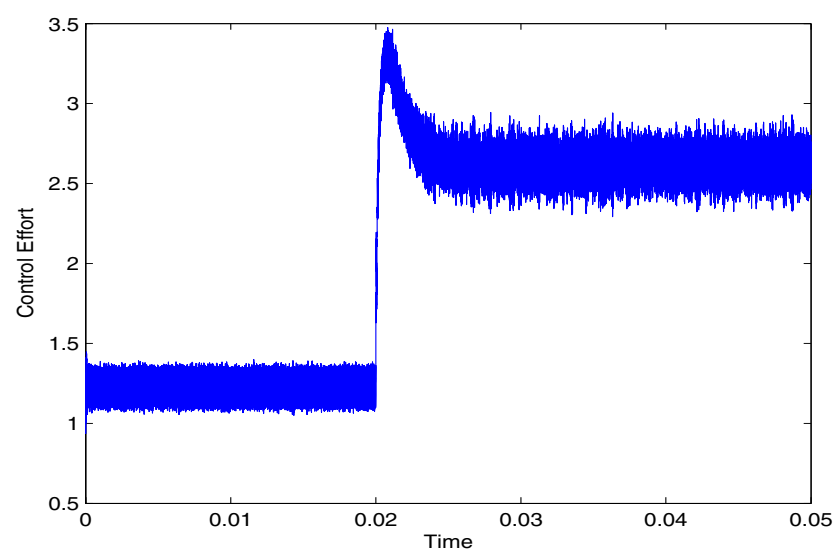

Fig. 11. Controller effort (10-operating points, $\Lambda=1$ )

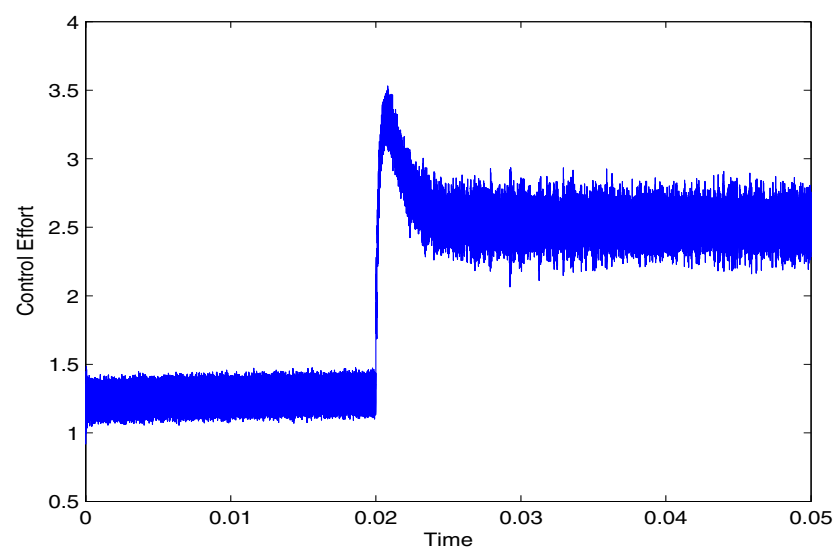

Fig. 12. Response of the micro-capacitor (10-operating points, $\Lambda=20$ ) 
From a numerical point of view, the satisfaction of a large number of inequalities in equation (11) is quite demanding. Subsequently, the sensitivity of these inequalities with respect to the $\Lambda$-parameter, as well as the $Q$ and $R$-parameters is important. Large values of $\Lambda$ will most likely result in a faster closed-loop system; however this poses stringent requirements on the satisfaction of these inequalities as $M$ increases. From a control point of view, a large number of operating points is desired while at the same time using a large number of $\Lambda$.

Figure 13 presents the maximum allowable $\Lambda, \Lambda \in$ $[0,100]$ for different number $M$ of operating points and various values of $Q$ and $R,(Q=100 R)$. From the presented results: i) if $M \rightarrow 1, \Lambda^{\max } \rightarrow 85$, and ii) if $M \rightarrow 10$, $\Lambda^{\max } \in[0,65]$ depending on the values of $Q$ and $R$.

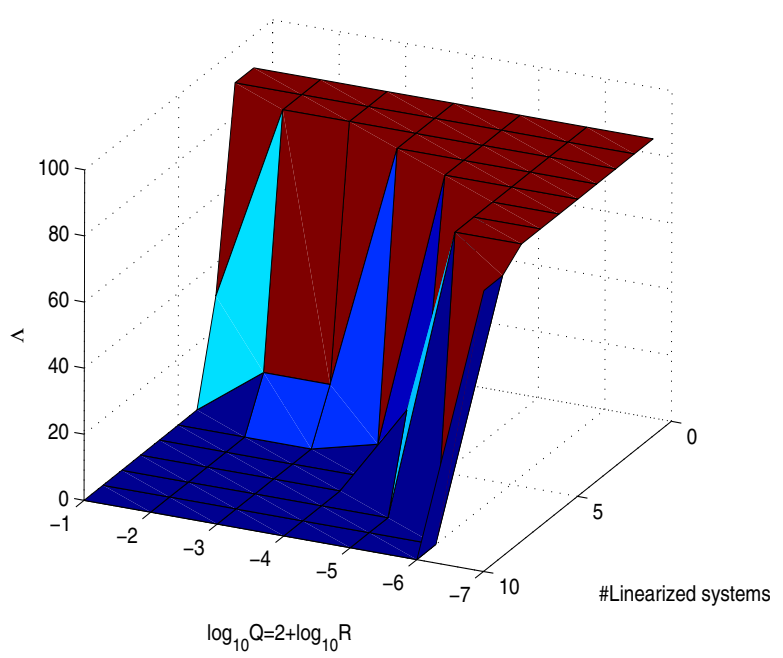

Fig. 13. Dependence between $Q, R, M$ and $\Lambda$ selection

\section{CONCLUSIONS}

In this article an intelligent controller has been designed for a micro-actuator system. The controller consists of: a) a feedforward portion, b) a PID-controller, and c) an intelligent prefilter. The parameters of the feedback controller are tuned via the usage of LMIs while the cutoff of the IPF is tuned in an ad-hoc manner suited for the nonlinear nature of the $\mu-\mathrm{A}$. The efficiency of the suggested scheme, as well its sensitivity on certain parameters is tested in a series of simulation studies. In future works, research studies will be conducted on a trade off, between the overshoot of the response and the magnitude of the noise in the corresponding control signal.

\section{REFERENCES}

[1] E. Lyshevski, "Microelectromechanical systems: Motion control of microactuators.," in IEEE/ASME Transactions on Mechatronics Proceedings of the IEEE Conference on Decision and Control, 1998.

[2] A. Menciassi and A. Eisinberg and I. Izzo and P. Dario, "From "macro" to "micro" Manipulation: Models and Experiments," in IEEEASME Transactions on Mechatronics, vol. 9, pp. 311-320, 2004.

[3] M. Madou, Fundamentals of Microfabrication. CRC PRESS, Boca Raton, FL, 1997.
[4] M. Sitti, "Survey of Nanomanipulation Systems," IEEENanotechnology Conference, pp. 75-80, November 2001.

[5] H. Ishihara, F. Arai, and T. Fukuda, "Micro mechatronics and micro actuators," IEEE/ASME Transactions on Mechatronics, vol. 1, pp. 6879, March 1996.

[6] A. Lee, C. McConaghy, G. Sommargren, P. Krulevitch, and E. Campbell, "Vertical - actuated electrostatic comb drive with in situ capacitive position correction for application in phase shifting diffraction interferometry," Journal of Microelectromechanical Systems, vol. 12, pp. 960-971, December 2003.

[7] H. Liu, B.Lu, Y. Ding, Y. Tang, and D. Li, "A motor - piezo actuator for nano - scale positioning based on dual servo loop and nonlinearity compensation," Journal of Micromechanics and Microengineering, vol. 13, pp. 295-299, March 2003.

[8] H. Zhang, A. Laws, V. Bright, K. Gupta, and Y. Lee, "MEMS variable - capacitor phase shifters Part I: Loaded - line phase shifter," International Journal of RF and Microwave Computer - Aided Engineering, vol. 13, pp. 321-337, July 2003.

[9] E. Lyshevski, "Micro-electromechanical systems: Motion control of micro-actuators," Proceedings of the IEEE Conference on Decision and Control, 1998.

[10] S. Hong, V. Varadan, and V. Varadan, "Implementation of coupled mode optimal structural vibration control using approximated eigenfunctions," Smart Material Structures, vol. 7, pp. 63-71, 1998.

[11] M. Zarubinskaya and W. Horssen, "On the free vibrations of a rectangular plate with two opposite sides simply supported and the other sides attached to linear springs," report 03-09, DELFT University of Technology, 2003.

[12] A. Tzes, G. Nikolakopoulos, L. Dritsas, and Y. Koveos, "Multiparametric $H_{\infty}$ control of a $\mu$-actuator," In the Proceedings of the 2005 IFAC World Congress, (Prague, Czech), no.4455, July 2005.

[13] M. Ge, M. Chiu, and Q. Wang, "Robust PID controller Design via LMI approach," Journal of Process Control, vol. 12, pp. 3-13, 2002.

[14] J. VanAntwep and R. Braatz, "A tutorial on linear and bilinear Matrix Inequalities ," Journal of Process Control, vol. 10, pp. 363-385, 2000.

[15] K. Narendra, J. Balakrishnan, and M.K.Ciliz, "Adaptation and Learning using multiple models, switching and tuning," IEEE Control Systems Magazine, vol. 15, pp. 37-51, 1995.

[16] Y. Cheng and C. Yu, "Nonlinear process control using multiple models: relay feedback approach ," Industrial Eng.Chem., vol. 39, pp. 420-431, 2000

[17] C. Chen, "A simple method for on-line identification and controller tuning," AIChE, vol. 35, pp. 2037-2039, 1989. 